\title{
Organic solar cell based on photosystem I pigment-protein complex, fabrication and optimization
}

\author{
Amirbahador Zeynali a, Talieh Sadat Ghiasi a, c, Gholamhossein Riazi ${ }^{\text {b }}$, Rasul Ajeian ${ }^{\text {a, * }}$ \\ a Thin-Films Laboratory, Department of Physics, Iran University of Science and Technology, Tehran, Iran \\ ${ }^{\mathrm{b}}$ Neuro-Organic Laboratory, Institute of Biochemistry and Biophysics (I.B.B), University of Tehran, Tehran, Iran \\ ${ }^{\mathrm{c}}$ Zernike Institute for Advanced Materials, University of Groningen, Groningen, The Netherlands
}

\section{A R T I C L E I N F O}

\section{Article history:}

Received 12 June 2017

Received in revised form

9 September 2017

Accepted 16 September 2017

Available online 19 September 2017

\section{Keywords:}

Hybrid solar cell

Photosystem I

Tyrosine

Fullerene-C60

\begin{abstract}
A B S T R A C T
The trends of using biological materials in electronic devices have made great developments in the last few years. Furthermore, the appealed cost features of organic semiconductors represent a bright lowcost, environment compatible, and efficient future for bio \& nanotechnologies, especially Bio-organic solar cells which may consider as a noteworthy option for photovoltaic applications. Here, we report a novel single junction organic solar cell based on photosystem I pigment-protein complex. The complex which operated either as photosensitizer and charge generator compound, surprisingly. Photosystem I complexes were extracted from young spinach leaves and used as the active layer of the intended solidstate solar cell device, subsequently. After the characterization of the final cell, our photovoltaic system showed the current density of $3470 \mu \mathrm{A} \mathrm{cm}^{-2}$ which realizes as a notable approach in between photosystem- I based energy conversion systems.
\end{abstract}

๑) 2017 Elsevier B.V. All rights reserved.

\section{Introduction}

Organic semiconductors (OSCs) used in optoelectronic applications instead of conventional rare, high cost, and pollutant inorganic compounds have already initiated the substantial interest in the scientific communities [1]. In general, the tunable mechanical and photo-electric properties of organic materials have opened the horizon with an enormous amount of possibilities to produce strong, flexible, and light-weight materials with the potential for mass production. As such, OSCs remain a topic of considerable interest for basic and applied research. Of course, this point of care leads to the generation of organic and hybrid inorganic/organicbased electronics. In respect to previously mentioned trend, LeBlanc and the co-workers [2,3] aligned with their earlier research, report articles which introduced a new bio-based material with interesting optoelectronic properties called photosystem I (PSI) pigment-protein complex.

Inevitably interaction of light in a wide variety of ways with the matter has been considered as a worthwhile and beneficial source of energy production in last decades. One of the most presented light-matter interaction has already known by the name of

\footnotetext{
* Corresponding author.

E-mail address: rasul_aj@iust.ac.ir (R. Ajeian).
}

photosynthesis mechanism. Oxygenic photosynthesis is an operation in which the absorbed light converted into chemical energy through cyclic activities of four multi-subunit membrane protein complexes: PSI, photosystem II (PSII), the cytochrome $b 6 \mathrm{f}$ complex, and F-ATPase in cyanobacteria, algae, and higher plants. PSI advantageously involved in both direct and indirect electron transfer reactions. On one hand, the light photons capture through its wellorganized peripheral antenna system consisting of pigment networks. Afterward, the excitation energy transfers to the reaction center (RC) where it is used in transmembrane electron transfer reaction. On the other hand, PSII capturing the light and oxidizes the water to produce oxygen and reduce membrane-embedded quinones. The reduced quinones are then utilized by the cytochrome $b 6 \mathrm{f}$ complex in the purpose of membrane proton gradient production to reduce donor site of the PSI, copper protein plastocyanin (PC). Afterward, the energy migrates through PSI pigment networks and reaches to $\mathrm{P700}$, a special chlorophyll pair, where electron generation take places [4,24].

Exhibiting a perfect conversion of absorbed photons to electrons (e.g. quantum efficiency) of nearly $100 \%$ [25] and notable negative redox potential, arguably introduces PSI as a natural photo-electric compound. This ability of PSI pigment-protein complex to harvest the energy of photons strongly depend on the spatial arrangement of protein subunits and relevant cofactors. Plant PSI made upon 17 
subunits and 193 non-covalently bound photochemical cofactors [5]. $7 \mathrm{~nm} \times 10 \mathrm{~nm} \times 15 \mathrm{~nm}$ PSI complex measured by electron microscopy [6] contains extraordinary electron transfer chains (ETCs) begin with $\mathrm{P}_{700}$, the first donor site, and two separated but almost similar branches, $A$ and $B$. The branches similarly contain $A_{c c}$ chlorophyll molecule (first acceptor/donor site), $A_{0}$ chlorophyll molecule (second acceptor/donor site), $A_{1}$ phylloquinone, (third acceptor/donor site), and three [4Fe-4S] iron-sulfur clusters called respectively $F_{X}, F_{A}$, and $F_{B}$.

Each photon energy h $v$ absorbed by antenna's pigment networks made of predominantly chlorophyll molecules and few $\beta$ carotenoids in approximately $10^{-15} \mathrm{~s}$. The absorbent energy excited peripheral pigment molecules, then the excitation energy migrates through internal pigments (e.g. resonance energy transfer), till they reach $\mathrm{P}_{700}$ site in the RC. Massive resonance energy harvested by chlorophyll special pair within the deep band gap and subsequently the work function difference between $\mathrm{P}_{700}$ and the first acceptor/ donor site, $A_{c c}$, caused generation of the electron to transfer through ETCs $[7,20]$.

Light-induced charge separation oxidizes the primary electron donor $\mathrm{P}_{700}$ chlorophylls a/a' heterodimer and $\mathrm{A}_{\mathrm{cc}}$ site with redox potential $\left(E_{R P}\right)$ of $430 \mathrm{mV}$, respectively. Then the second electron acceptor $A_{0}$ a chlorophyll monomer reduced ( $E_{R P}$ of $-1000 \mathrm{mV}$ ), then the electron transferred to $A_{1}\left(E_{R P}\right.$ of $-800 \mathrm{mV}$ ) then to $F_{X}\left(E_{R P}\right.$ of $-705 \mathrm{mV}), \mathrm{F}_{\mathrm{A}}\left(\mathrm{E}_{\mathrm{RP}}\right.$ of $-520 \mathrm{mV}$ ) and finally $\mathrm{F}_{\mathrm{B}}\left(\mathrm{E}_{\mathrm{RP}}\right.$ of $\left.-580 \mathrm{mV}\right)$ $[8,9]$.

This novel photovoltaic function of PSI has been recently applied in different optoelectronic devices. For example, PSI deposited on various conducting and semi-conducting material substrates like $\mathrm{Au}$ [10-13], ITO [14], Graphene derivatives [15], and $\mathrm{TiO}_{2}$ [16] for photocurrent generation purposes. The noteworthy approach toward fabricating a solid-state photovoltaic cell based on PSI is reported by Gordiichuk and co-workers [17]. There is a valuable review on PSI photovoltaics which maintains more detailed applications and records [18]. In this approach, our group previously has fabricated two different photovoltaic system based on PSI pigmentprotein complex extracted from young spinach leaves, an electrolyte cell and a solid-state one, which prepares additionally a comparison situation. Here we report our latest solid-state bio-organic solar cell based on PSI pigment-protein complex.

\section{Experimental}

As shown in Fig. 1, the intended solar cell stack contains amino acidic substrate, PSI multi-layer, a rough and porous interlayer of fullerene-C60, Indium tin oxide (ITO) as the anode, and the cathode made of thin film of gold.

\subsection{Isolation of PSI complex from spinach leaves}

Preparation of PSI pigment-protein complex [19]. Fresh leaves of spinach (Spinacia oleracea L.) were purchased from the local market and then prechilled in the dark overnight. The leaves were homogenized in a buffer containing sucrose $(0.3 \mathrm{M}$, Sigma-Aldrich, 99.5\%), NaCl (15 mM, EMD Millipore) and tricine (30 mM, SigmaAldrich, 99\%)- $\mathrm{NaOH} \mathrm{pH}$ 7.8. The slurry was filtered through four layers of cheesecloth and centrifuged for $2 \mathrm{~min}$ at $2000 \mathrm{~g}$. The resulting chloroplasts were washed twice in a hypotonic buffer containing EDTA ( $5 \mathrm{mM}$, Sigma-Aldrich) and $5 \mathrm{mM}$ tricine- $\mathrm{NaOH}$ $\mathrm{pH}$ 7.8. Unstacked membranes were isolated by centrifugation at $25000 \mathrm{~g}$ for $10 \mathrm{~min}$ and suspended in a buffer containing $0.3 \mathrm{M}$ sucrose and $30 \mathrm{mM}$ tricine- $\mathrm{NaOH} \mathrm{pH} 7.8$.

Spinach thylakoids containing $6 \mathrm{mg}$ Chl. $\mathrm{mL}^{-1}$ were solubilized in Triton X-100 (4.8\% w/v, EMD Millipore). After stirring for $15 \mathrm{~min}$, the sample was centrifuged for $15 \mathrm{~min}$ at $15000 \mathrm{~g}$. The complex was further purified by diethyl aminoethyl (DEAE)-cellulose (Merck Millipore) column chromatography with an elution buffer containing $20 \mathrm{mM}$ tricine- $\mathrm{NaOH} \mathrm{pH} 7.8,0.03 \% \mathrm{DM}$. All steps were performed at $4^{\mathrm{oC}}$ under dim light. The collected solutions from the column contain PSI complexes were then dialyzed.

\subsection{Fullerene-C60 preparation}

Fullerene-C60 (Sigma-Aldrich, 99.5\%) in solution phase, for spin-coating, were prepared with toluene (EMD Millipore) as the initial solvent and deionized water as the second solvent. $2.8 \mathrm{mg} / \mathrm{ml}$ is the solubility of fullerene C60 in toluene [26]. The measured volume of toluene was added to $4 \mathrm{mg}$ C60 powder. After a few second of stirring, a solution with purplish color appeared, Fig. 2a. The solution was stirred on a magnetic stirrer in a beaker for about $20 \mathrm{~min}$ to reach a homogenized solution. Afterward, the amount of $40-50 \mathrm{ml}$ deionized water was added to the system, Fig. 2b. After a while with respect to heating and using ultrasonic stirrer, toluene was evaporated and the fullerene-C60 was dispersed in deionized water made a brownish solution. The final solution were filtered through filter paper (Whatman $25 \mathrm{~mm}$ ) to lose large aggregates. Fig. 2c.

\subsection{Tyrosine amino acid}

Suspension of tyrosine (L-Tyrosine, Sigma-Aldrich, 99\%) in deionized water, for dip-coating, were prepared with just deionized water as the solvent. The precise amount of tyrosine (depend on intended area of coating) powder were added to deionized water. The complex was stirred well to minimize the size of tyrosine needles.

\subsection{Bio-organic solar cell fabrication}

Prior to device fabrication, the ITO-coated glasses (Sheet resistivity of $100 \Omega / \mathrm{sq}$ ) were cleaned with the standard procedure using distilled water, acetone, and isopropanol. The substrates were then masked with plastic tapes and treated with UV/O3 for $10 \mathrm{~min}$, respectively. The masked substrates were dipped in activated tyrosine suspension and rest for 2 days. Afterward, the samples were pulled out carefully and dried under clean-desk atmosphere. Deposited layer of tyrosine was soaked gently in deionized water to remove any unbounded colloids. After a mild drying processing, the solution containing PSI were deposited through spin-coating. In the next step, fullerene-C60 were deposited through spin-coating, subsequently. The samples were transferred to a sputtering machine contains gold cathode. Finally, the device were finished with 30-40 nm layer of gold deposited via sputtering under the condition of 150 mbar for about $300 \mathrm{~s}$. The masks were carefully rejected and cells with the active area of $50 \mathrm{~mm}^{2}$ were obtained.

\subsection{Characterization}

Device performance measurements were done using solar simulator contains metal halide lamp of AM 1.5G spectrum with $80 \mathrm{mWcmm}^{-2}$ calibration. Cross section and surface morphologies of the layers were studied by scanning electron microscopy (SEM, Tescan Vega/II). Hitachi-CF16RX and SigmaII-3-30 K centrifuge machines were used during protein extraction. Spectrophotometric characterizations were recorded via both UV-Vis Carry 100 Bio and ELIZA power wave xs2.

\section{Results and discussions}

Although this quest provides advancements in organic-based 


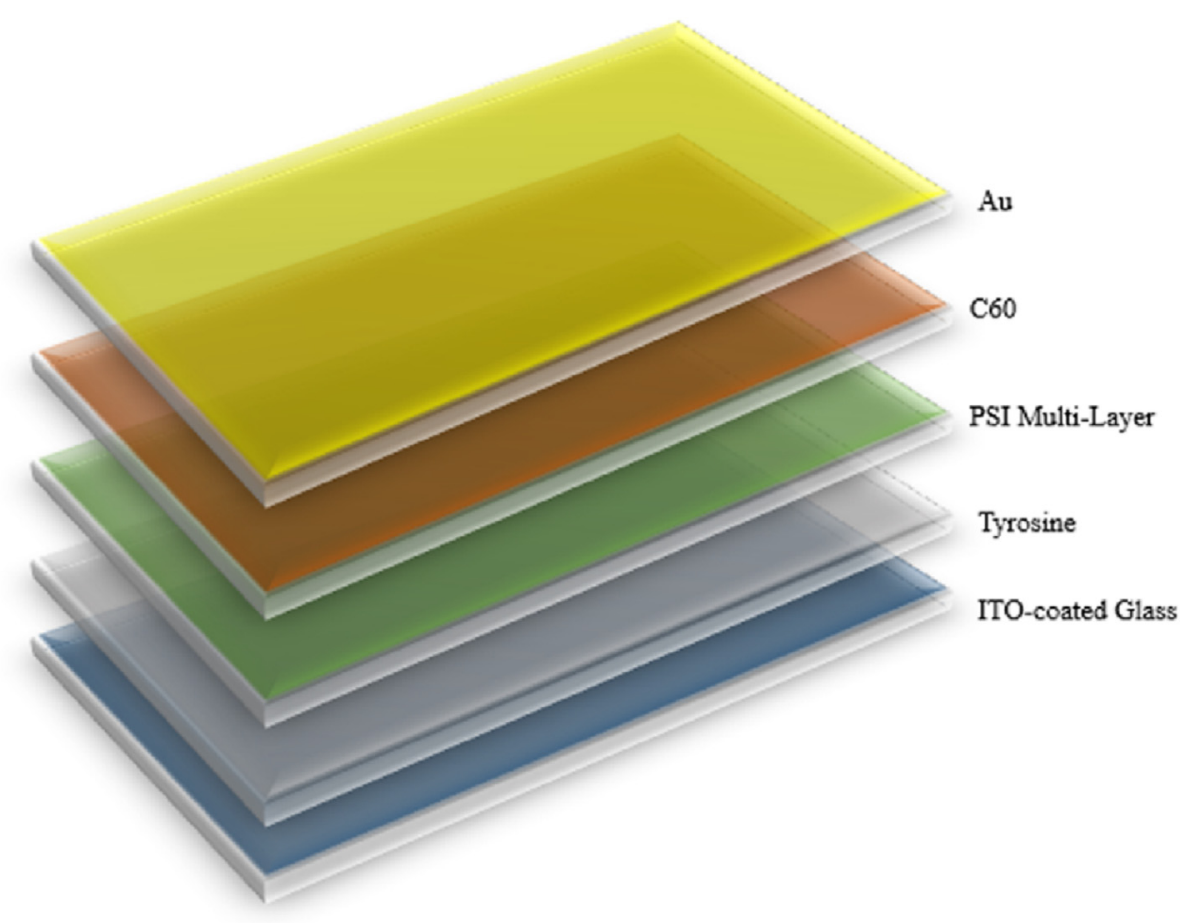

Fig. 1. Bio-organic solar cell stack (ITO/Tyrosine/PSI/C60/Au).

(a)

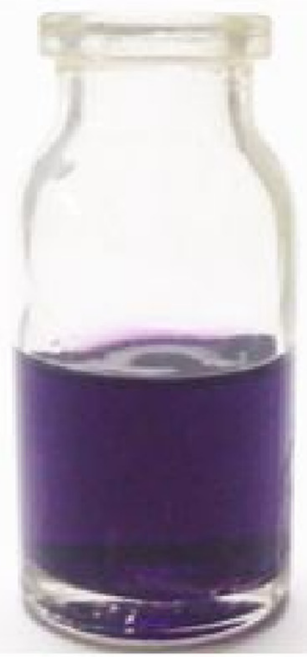

(b)

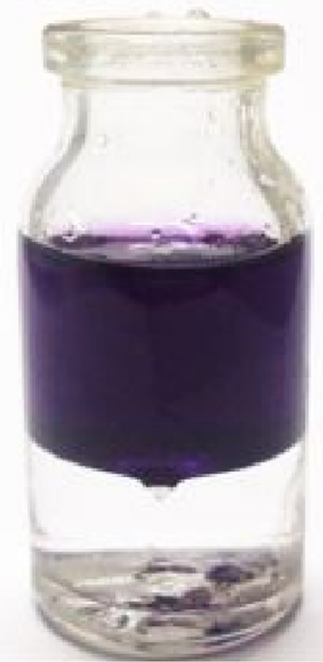

(c)

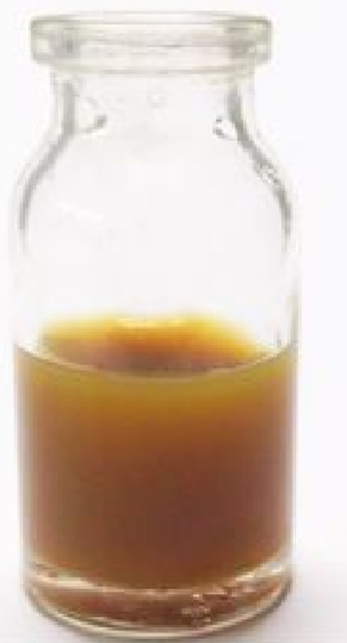

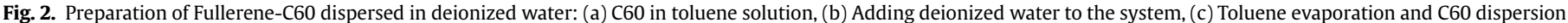
in deionized water.

energy conversion devices, it suffers from the relatively hard compatibility of biological compounds in the non-inherent environments, for example in this study solid-state solar cell. Therefore, some efforts were organized in order to optimize the functionalities of the solid-state bio-organic solar cell contains PSI complex multilayer. In this approach, three suggestions were applied to optimize the device performance.

(i) Regarding with the importance of PSI orientation for charge carriers transport through energy levels, a layer made of tyrosine amino acids was employed as a buffer layer between
ITO and PSI. The partially positive activated surface of ITO was attracted to the free radical oxygen $(-\mathrm{OH})$ of Phenolic end. The Amine $(-\mathrm{NH} 2)$ side chains were attached with free oxygen radical of Carboxyl. Subsequently, the array of tyrosine molecules (Poly-tyrosine) appeared on ITO substrate with free Phenolic ends, Fig. 3.

(ii) The micro-needle shape of tyrosine molecules preferably act as a rough surface. Therefore, the cross section for photons impact is raised which arguably has a positive effect on the efficiency of the cell [27]. Fig. 4a-b. 

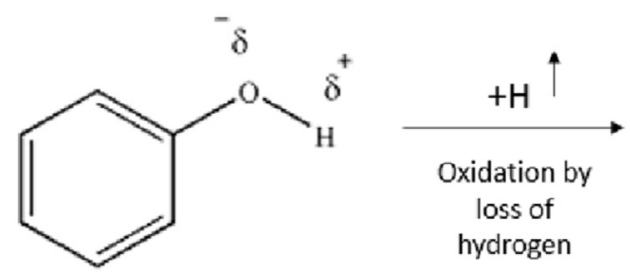<smiles>Oc1ccccc1</smiles>

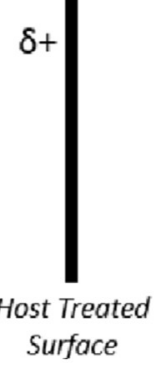

Fig. 3. Poly-tyrosine molecules: negative and free phenolic terminals attract toward partially positively charged ITO.
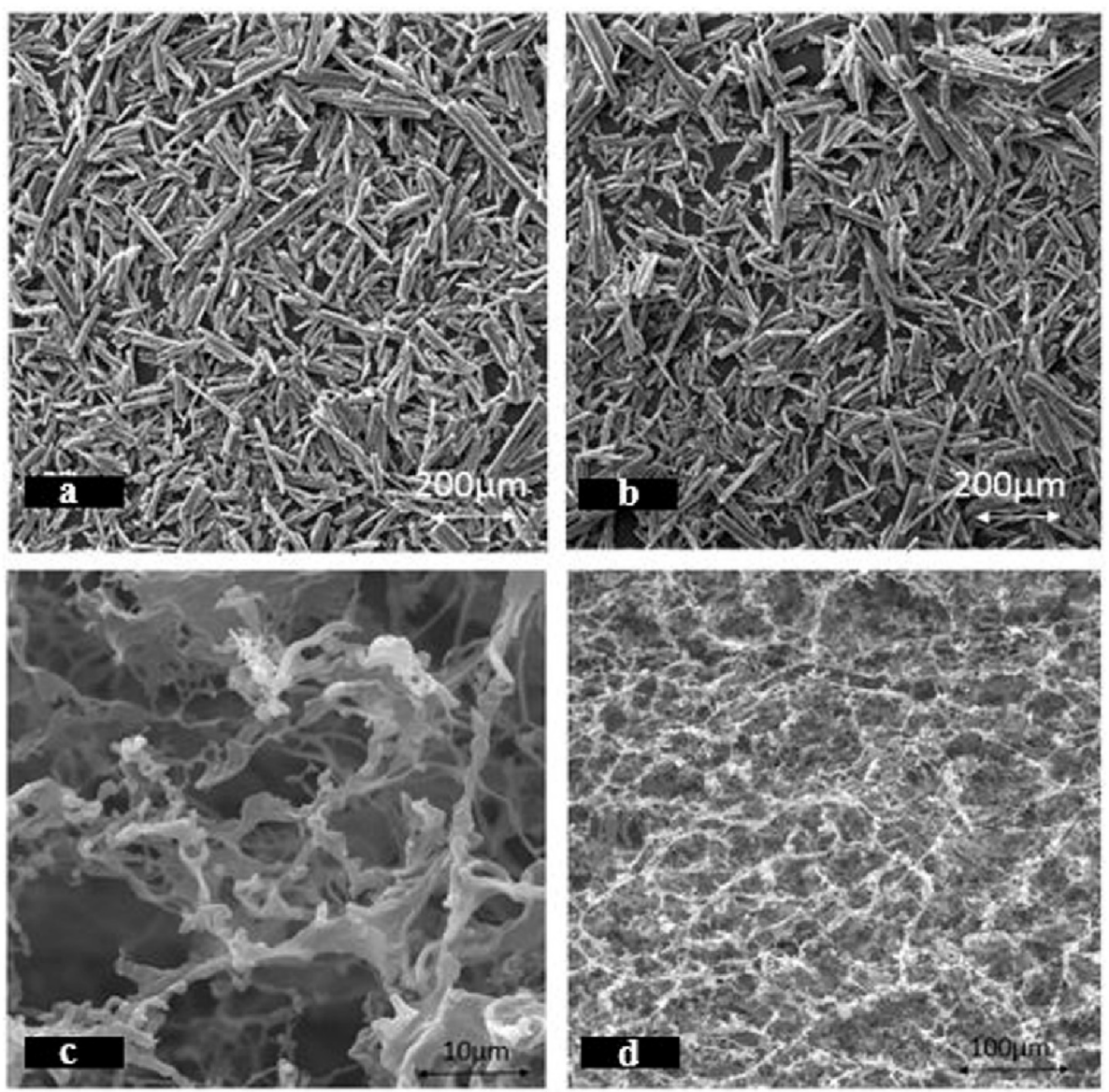

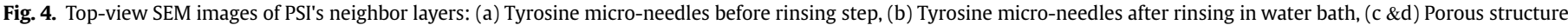
of Fullerene-C60 layer prepared under treatment of C60 dispersion in deionized water.

(iii) Dispersed C60 demonstrated extraordinary Nano-strings structure, Fig. 4c-d. Nano-strings porous property was prevented the PS1 layer in losing lots of its moisture. In addition, the PSI sensitive layer was protected via this layer from defects caused by deposition of the gold electrode layer. We managed our experimental works in the way which the PSIs sense less environmental damages, we avoid using the Physical Vapor Deposition (PVD) or similar techniques for deposition of $\mathrm{C60}$ which is all the known and homogeneous ways, and in addition avoid the post-phase annealing steps.

In Fig. 5, the selected results of chromatography were demonstrated. Observing absorption spectrum of each output through chromatography steps helped to find the richest solution in the PSI complex. Regarding with environmental condition of the spinach plant, the absorption peak for PSI varies from $670 \mathrm{~nm}$ to $680 \mathrm{~nm}$ [20]. 


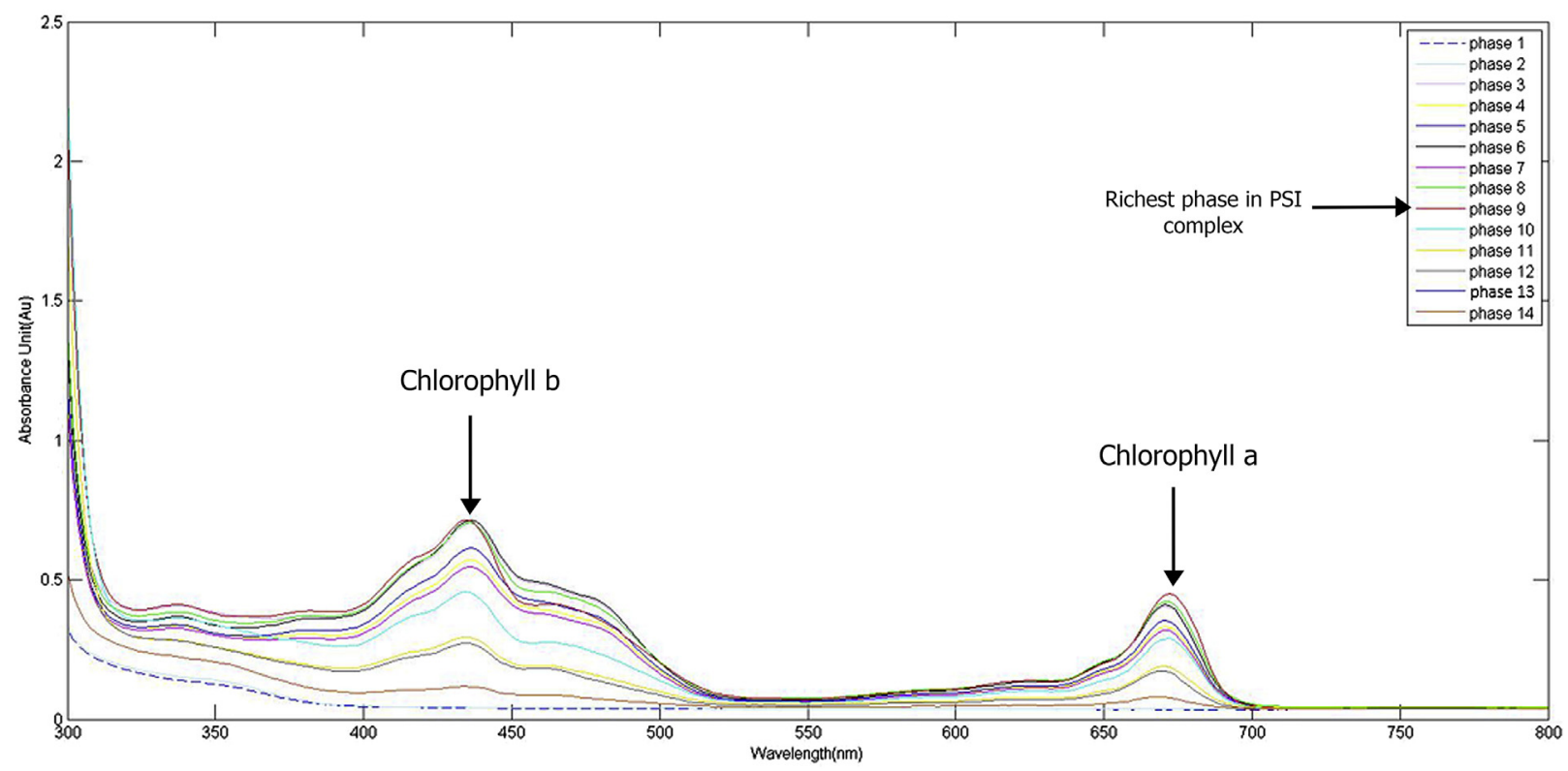

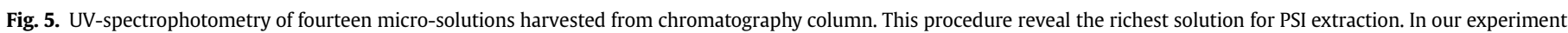
phase 9 shows the highest peak in range of chlorophyll $a$ absorption spectrum.

According to energy diagram, Fig. 6, the device was fabricated. The results and the outputs of the bio-organic solar cell are demonstrated in Table 1. This collation demonstrates the importance of inter-layer for PSIs to make potential links with electrodes in the purpose of efficient charge transport.

Fig. 7 shows the light/dark activity of the device without PSI. Regarding with the deposition of PSIs to act as an active layer of the device. I-V curve of the final cell, and the comparison situation in Fig. 8 demonstrate the activity of bio-organic solid state solar cell based on PSI pigment-protein complex.

To the best of our knowledge, $3470 \mu \mathrm{A} / \mathrm{cm}^{2}$ current density ( $\mathrm{J}_{\mathrm{SC}}$ )
Table 1

Photovoltaic parameters of fabricated device presented with two different cell strategies.

\begin{tabular}{lllll}
\hline Structure & $\mathrm{V}_{\mathrm{OC}}(\mathrm{V})$ & $\mathrm{JsC}\left(\mathrm{mA} \mathrm{cm}^{-2}\right)$ & $\mathrm{FF}(\%)$ & $\mathrm{PCE}(\%)$ \\
\hline Tyrosine + PSI & 0.03 & -0.12 & 29 & 0.001 \\
Tyrosine + PSI + C60 & 0.36 & -3.47 & 33 & 0.517 \\
\hline
\end{tabular}

of our bio-organic solar cell could be a remarkable achievement between identical reports on this topic. The latest experiment in this category is addressed by Gizzie and co-workers [21].

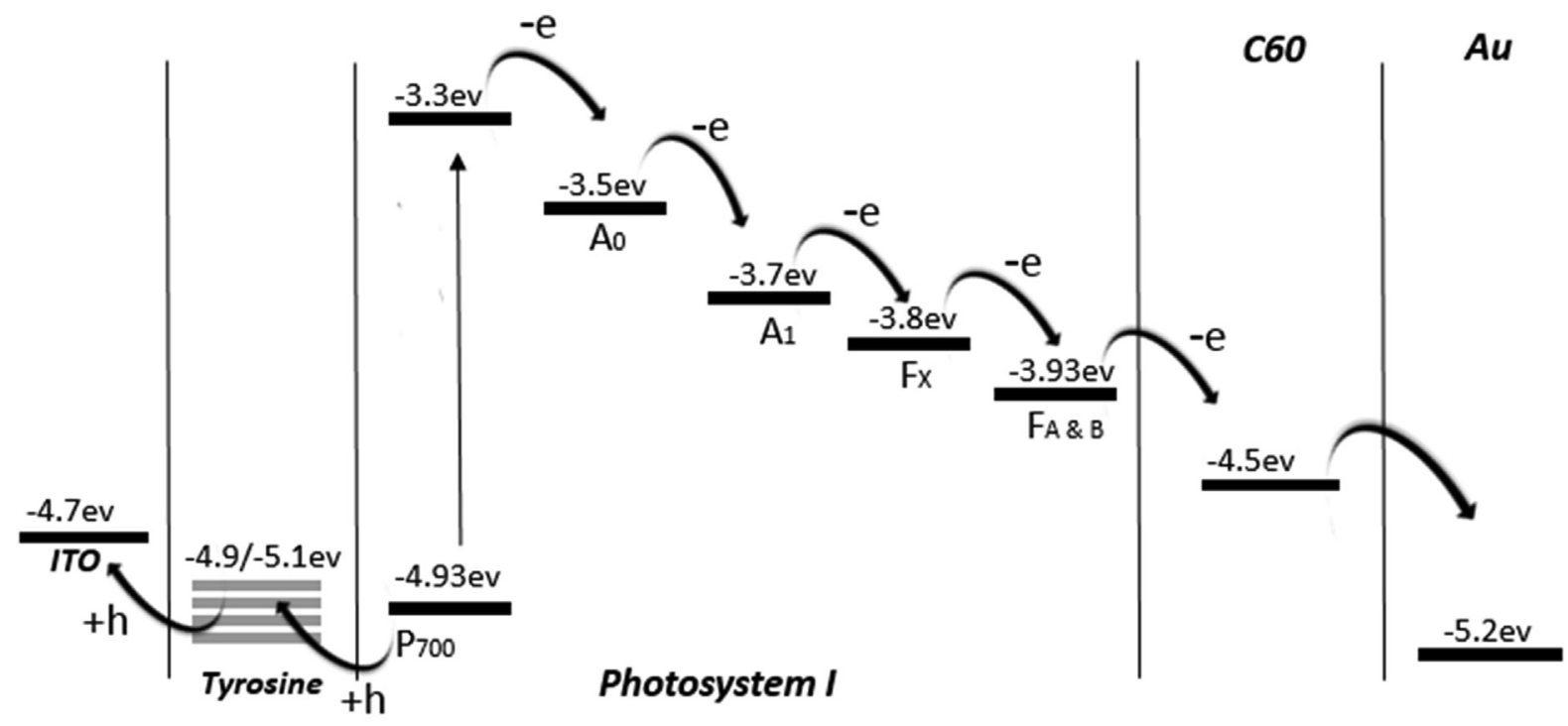




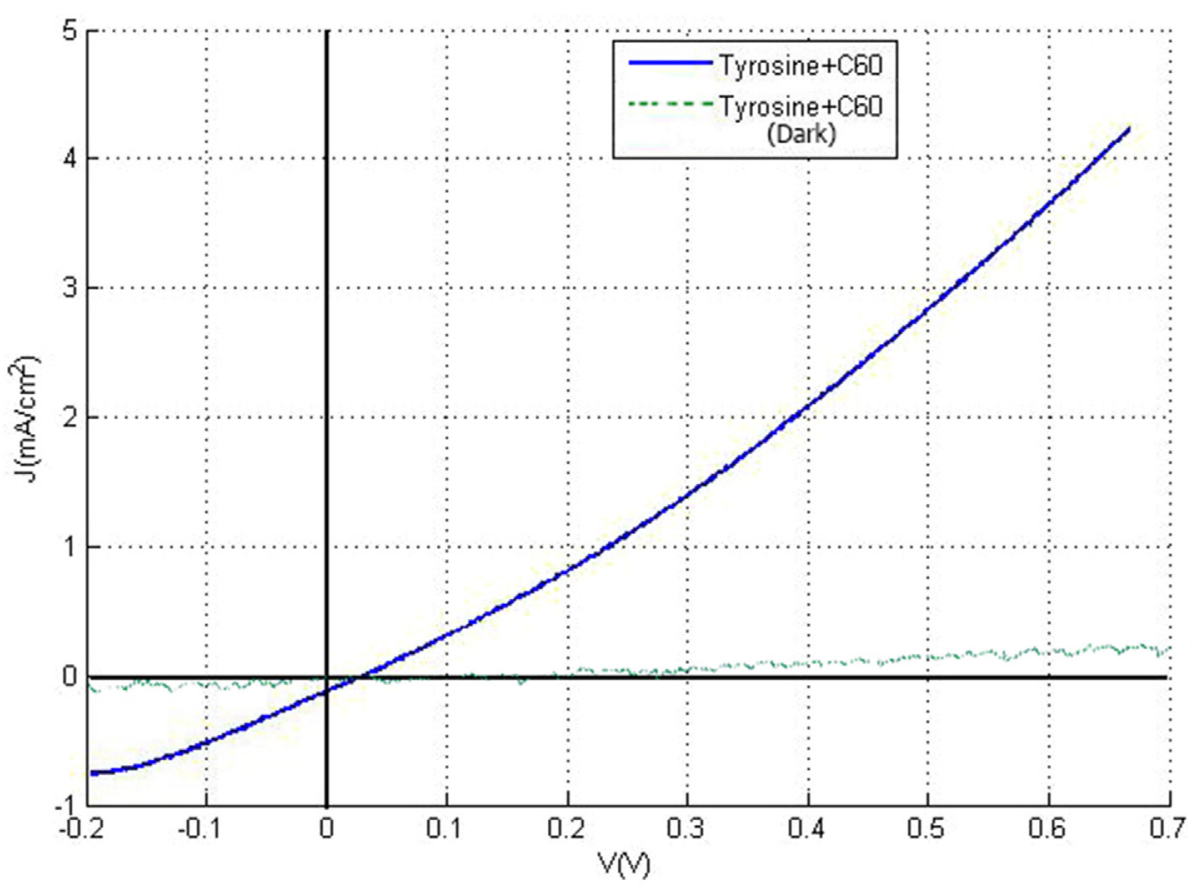

Fig. 7. I-V characteristic of the cell without the PSI active layer.

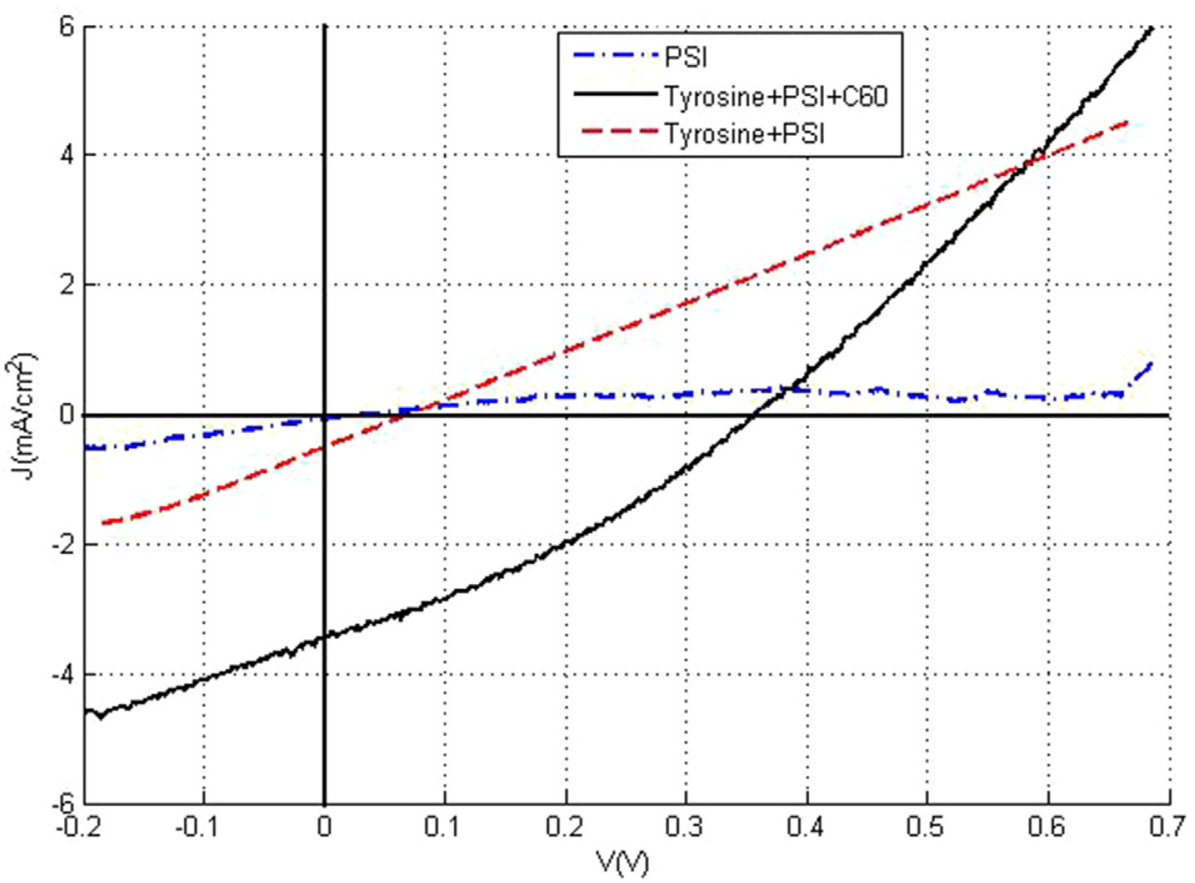

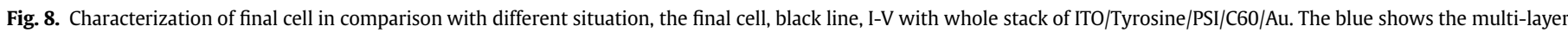
of PSI complex sandwiched between ITO and Au cathodes, and the red line is a demonstration of the cell in absence of C60 inter-layer.

As we know, the solar cell is basically a diode connected with a current source in parallel. The parasitic resistors i.e. Series $\left(R_{S}\right)$ and Shunt $\left(\mathrm{R}_{\mathrm{SH}}\right)$ are also incorporated into the equivalent circuit to represent different parasitic effects inside the solar cell $[22,23]$. The approximate value of $R_{S}$ and $R_{S H}$ can also be extracted from I-V curve. Under ideal solar cell conditions, $\mathrm{R}_{S}$ should be as small as possible while $\mathrm{R}_{\mathrm{SH}}$ should be too large. With respect to this, the I-V curve of our device suffers from low shunt resistance which means the device has had current leakage. In our point of view, the current leakage happens with the situation which demonstrated in Fig. 9. The difference between two PSI heads (P700 and the 4Fe-4S cluster) in the charge density and polarity slightly made each PSI molecule to act as a dipole. Regarding this, we believe that the optimize orientation of the PSI molecules aligned in the direction the cell structure would raise the PCE, subsequently. The poor alignment of PSI molecules causes damping of the charge carriers. 
(a)

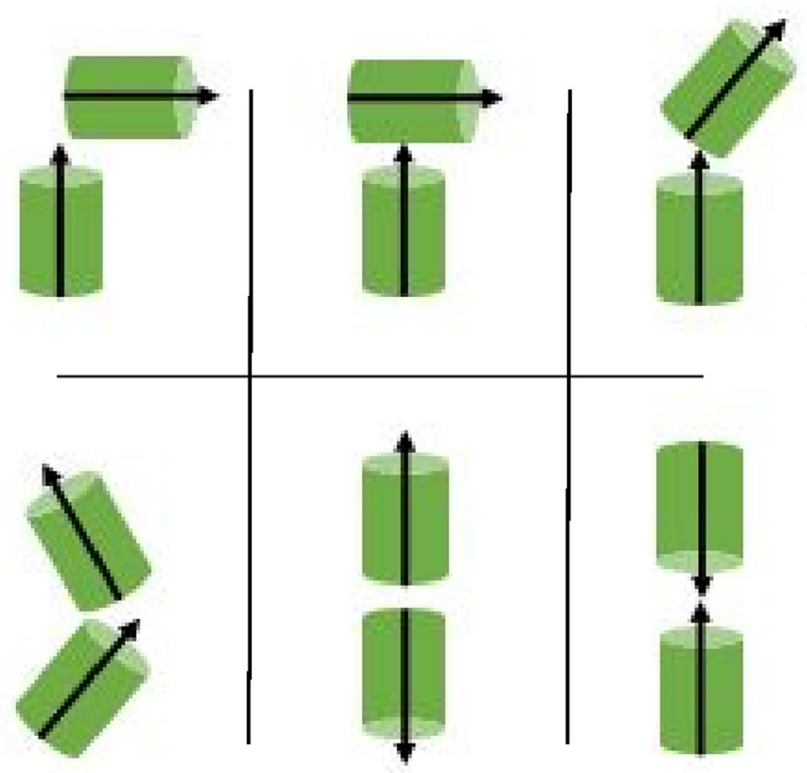

(b)

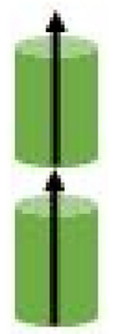

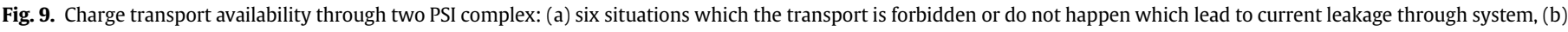
an available state for efficient charge transport.

Therefore, with multi-layer deposition of PSIs, it is really important to control the assembly of PSI molecules. This would be the next step for our further investigations on this topic. For topographic purposes and analyzing the conductivity of the PSI layer (bulk), the Atomic Forced Microscopy were assisted. The PSI-bulk layer was deposited on the silica substrate under the same condition of the real experimental cells. Fig. 10.

\section{Conclusion}

All in all, the bio-organic solid-state solar cell based on PSI pigment-protein complex was fabricated and optimized regard with PSI new isolation method, compatible inter-layers, effective interface area, heat-free deposition techniques, and low vacuum situations. Although the Power Conversion Efficiency (PCE) of $0.51 \%$
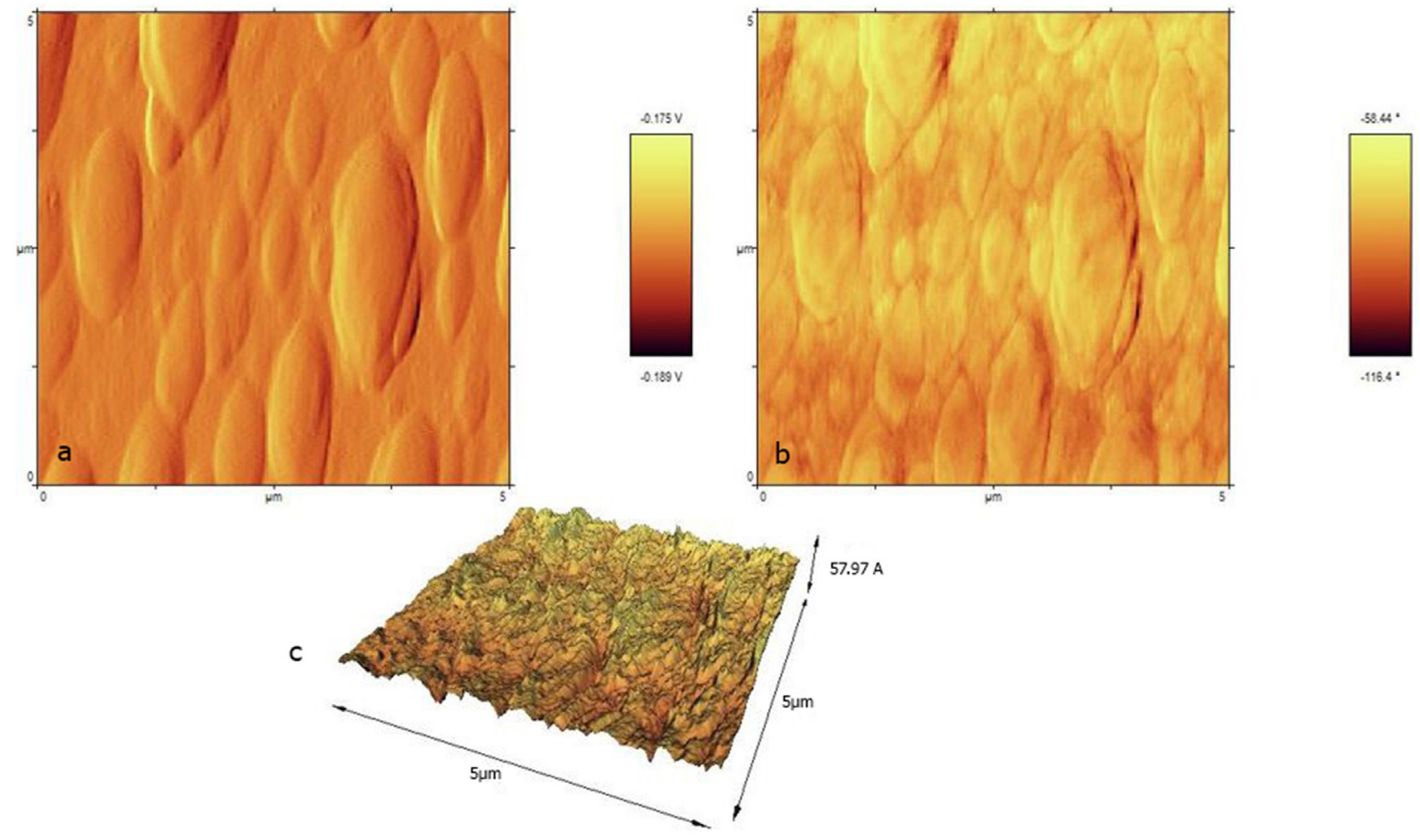

Fig. 10. AFM images of PSI-bulk layer on the silica substrate: (a) topography of 100-amplitude probing (b) backward analyzing (c) 3D-plate of same surface. 
is very low however, it promises a bright future for solar energy conversion systems with more environment compatibility and low costs conditions. For further studies, it is important to control the assembly of PSI layer by layer or in advanced situations molecule by molecule, design a perfect device architecture, matchable energylevels, and try engineering the PSI's ETCs for better charge carrier mobility.

\section{References}

[1] O. Ostroverkhova, Organic optoelectronic materials: mechanisms and applications, Chem. Rev. 116 (22) (2016) 13279-13412.

[2] E. Darby, G. LeBlanc, E.A. Gizzie, K.M. Winter, G.K. Jennings, D.E. Cliffel, Photoactive films of photosystem I on transparent reduced graphene oxide electrodes, Langmuir ACS J. Surf. Colloids 30 (29) (2014) 8990-8994.

[3] G. LeBlanc, E. Gizzie, S. Yang, D.E. Cliffel, G.K. Jennings, Photosystem I protein films at electrode surfaces for solar energy conversion, Langmuir ACS J. Surf. Colloids 30 (37) (2014) 10990-11001.

[4] J. Barber, The structure of photosystem I, Nat. Struct. Biol. 8 (7) (2001) $577-579$.

[5] A. Busch, Composition and structure of photosystem I in the moss Physcomitrella patens, J. Exp. Bot. 64 (10) (May 2013) 2689-2699.

[6] E.J. Boekema, R.M. Wynn, R. Malkin, The structure of spinach Photosystem I studied by electron microscopy, Biochim. Biophys. Acta (BBA) - Bioenergetics 1017 (1) (May 1990) 49-56.

[7] J.H. Golbeck, Photosystem I: the Light-driven Plastocyanin: Ferredoxin Oxidoreductase, Springer-Verlag, Dordrecht, 2006. New York.

[8] Stefano Santabarbara, Peter Heathcote, Michael C.W. Evans, Modelling of the electron transfer reactions in Photosystem I by electron tunnelling theory: the phylloquinones bound to the PsaA and the PsaB reaction centre subunits of PS I are almost isoenergetic to the iron-sulfur cluster FX, Biochim. Biophys. Acta (BBA) - Bioenergetics 1708 (3) (2005) 283-310.

[9] A.Y. Semenov, M.D. Mamedov, S.K. Chamorovsky, Electrogenic reactions associated with electron transfer in Photosystem I, in: Advances in Photosynthesis and Respiration, Springer Nature, May. 2006, pp. 319-338.

[10] Ida Lee, James W. Lee, Elias Greenbaum, Biomolecular electronics: vectorial arrays of photosynthetic reaction centers, Phys. Rev. Lett. 79 (17) (1997) 3294-3297.

[11] Madalina Ciobanu, Helen A. Kincaid, Vivian Lo, Albert D. Dukes, G. Kane Jennings, David E. Cliffel, Electrochemistry and photoelectrochemistry of photosystem I adsorbed on hydroxyl-terminated monolayers, J. Electroanal. Chem. 599 (1) (2007) 72-78.

[12] C.J. Faulkner, S. Lees, P.N. Ciesielski, D.E. Cliffel, G.K. Jennings, Rapid assembly of photosystem I monolayers on gold electrodes, Langmuir ACS J. Surf. Colloids 24 (16) (2008) 8409-8412.

[13] P.N. Ciesielski, A.M. Scott, C.J. Faulkner, B.J. Berron, D.E. Cliffel, G.K. Jennings,
Functionalized nanoporous gold leaf electrode films for the immobilization of photosystem I, ACS Nano 2 (12) (2008) 2465-2472.

[14] Omer Yehezkeli, Ran Tel-Vered, Dorit Michaeli, Rachel Nechushtai, Itamar Willner, Photosystem I (PSI)/Photosystem II (PSII)-Based photobioelectrochemical cells revealing directional generation of photocurrents, Small 9 (17) (2013) 2970-2978.

[15] D. Gunther, G. LeBlanc, D. Prasai, J.R. Zhang, D.E. Cliffel, K.I. Bolotin, G.K. Jennings, Photosystem I on graphene as a highly transparent, photoactive electrode, Langmuir ACS J. Surf. Colloids 29 (13) (2013) 4177-4180.

[16] V.V. Nikandrov, Ya. V. Borisova, E.A. Bocharov, M.A. Usachev, G.V. Nizova, V.A. Nadtochenko, E.P. Lukashev, B.V. Trubitsin, A.N. Tikhonov, V.N. Kurashov M.D. Mamedov, A. Yu. Semenov, Photochemical properties of photosystem 1 immobilized in a mesoporous semiconductor matrix, High Energy Chem. 46 (3) (2012) 200-205.

[17] Pavlo I. Gordiichuk, Gert-Jan A.H. Wetzelaer, Dolev Rimmerman, Agnieszka Gruszka, Jan Willem de Vries, Manfred Saller, Daniel A. Gautier, Stefano Catarci, Diego Pesce, Shachar Richter, Paul W.M. Blom, Andreas Herrmann, Solid-state biophotovoltaic cells containing photosystem I, Adv. Mater. 26 (28) (2014) 4863-4869.

[18] Khoa Nguyen, Barry D. Bruce, Growing green electricity: progress and strategies for use of Photosystem I for sustainable photovoltaic energy conversion, Biochim. Biophys. Acta (BBA) - Bioenergetics 1837 (9) (2014 $1553-1566$

[19] X. Qin, W. Wang, K. Wang, Y. Xin, T. Kuang, Isolation and characteristics of the PSI-LHCI-LHCII Supercomplex under high light, Photochem. Photobiol. 87 (1) (Nov. 2010) 143-150.

20] P.E. Jensen, R. Bassi, E.J. Boekema, J.P. Dekker, S. Jansson, D. Leister, C. Robinson, H.V. Scheller, Structure, function and regulation of plant photosystem I, Biochim. Biophys. Acta 1767 (5) (2007) 335-352.

[21] Evan A. Gizzie, J. Scott Niezgoda, Maxwell T. Robinson, Andrew G. Harris, G. Kane Jennings, Sandra J. Rosenthal, David E. Cliffel, Photosystem I-polyaniline/TiO2 solid-state solar cells: simple devices for biohybrid solar energy conversion, Energy Environ. Sci. 8 (12) (2015) 3572-3576.

[22] N.S. Sariciftci, S.-S. Sun (Eds.), Organic Photovoltaics; Mechanism, Materials, and Devices (Optical Engineering), Taylor \& Francis, Boca Raton, FL, 2005.

[23] Organic Solar Cells; Theory, Experiment, and Device Simulation, Springer International Publishing, New York, NY, United States, 2014

[24] B. Ke, Photosynthesis Photobiochemistry and Photobiophysics, Kluwer Academic Publishers, Dordrecht, 2003.

[25] Agu Laisk, Vello Oja, Hillar Eichelmann, Luca Dall'Osto, Action spectra of photosystems II and I and quantum yield of photosynthesis in leaves in State 1, Biochim. Biophys. Acta (BBA) - Bioenergetics 1837 (2) (2014) 315-325.

[26] C.I. Wang, C.C. Hua, Solubility of C60 and PCBM in organic solvents, J. Phys Chem. B 119 (45) (2015) 14496-14504.

[27] Zaifei Ma, Zheng Tang, Ergang Wang, Mats R. Andersson, Olle Inganäs, Fengling Zhang, Influences of surface roughness of $\mathrm{ZnO}$ electron transport layer on the photovoltaic performance of organic inverted solar cells, J. Phys. Chem. C 116 (46) (2012) 24462-24468. 